\title{
Abordagens teórico-práticas e metodológicas na formação em enfermagem para a saúde mental
}

Theoretical-practical and methodological approaches in Nursing education for mental health care Abordajesteórico-prácticos y metodológicos enlaformación de enfermería para lasalud mental

\section{Fabiana Porto da Silva ${ }^{\mathrm{I}}$, Daiana Foggiato de Siqueira ${ }^{\mathrm{II}}$, Mara Regina Caino Teixeira Marchiori ${ }^{\mathrm{III}}$, Juliana Silveira Colomé ${ }^{\mathrm{IV}}$}

\begin{abstract}
Resumo: Objetivo: identificar as atividades curriculares teórico-práticas e metodológicas na formação do enfermeiro relacionadas à educação em saúde mental. Método: estudo documental, descritivo e exploratório, de abordagem qualitativa. Foi desenvolvido em dois Cursos de Graduação em Enfermagem de um município do interior do Estado do Rio Grande do Sul, sendo um deles de caráter público e outro, privado. Foram analisados os planos de ensino de disciplinas, segundo análise documental. Os dados foram coletados no segundo semestre de 2018. Resultados: as atividades curriculares teórico-práticas e metodológicas relacionadas à educação em saúde mental estão inseridas de modo articulado a outros saberes, mostrando que a dimensão teórica vem acompanhando as transformações nesse campo de saberes e práticas. Conclusão: as disciplinas contemplam abordagens coerentes com as situações sociais, econômicas e culturais, porém observam-se poucas informações sobre a dimensão metodológica, relacionada aos cenários de práticas para a educação em saúde mental.

Descritores: Educação em enfermagem; Instituições de ensino superior; Política de saúde, saúde mental; Sistema único de saúde
\end{abstract}

Abstract: Objective: to identify theoretical-practical and methodological curricular activities in the training of nurses related to mental health education. Method: it is a documentary, descriptive and exploratory study, with a qualitative approach. It was developed in two Undergraduate Nursing Courses in a city in the State of Rio Grande

\footnotetext{
${ }^{\text {I }}$ Enfermeira. Mestre em Ciências da Saúde e da Vida pela Universidade Franciscana. Docente do Curso de Enfermagem. Universidade Franciscana - UFN, Santa Maria, Rio Grande do Sul, Brasil. E-mail: fabianaporto54@gmail.com ORCID: https://orcid.org/0000-0001-5450-2602

${ }^{\text {II }}$ Enfermeira. Doutora em Enfermagem pela Universidade Federal de Santa Maria. Docente do Curso de Enfermagem. Universidade Federal de Santa Maria - UFSM, Santa Maria, Rio Grande do Sul, Brasil. E-mail: daianasiqueira@yahoo.com.br ORCID: https://orcid.org/0000-0002-8592-379x

III Enfermeira. Doutora em Ciências pela Universidade Federal do Estado de São Paulo. Docente dos Cursos de Enfermagem e Mestrado em Saúde Materno- Infantil. Universidade Franciscana - UFN, Santa Maria, Rio Grande do Sul, Brasil. E-mail: mara.marc@hotmail.com ORCID: https://orcid.org/0000-0001-9412-7755

IV Enfermeira. Doutora em Enfermagem pela Universidade Federal do Rio Grande - FURG. Docente dos Cursos de Enfermagem e Mestrado em Ciências da Saúde e da Vida. Universidade Franciscana - UFN, Santa Maria, Rio Grande do Sul, Brasil. E-mail: julianacolome@yahoo.com.br ORCID: https://orcid.org/0000-0002-8059-1482
} 
do Sul, one of which is public and the other, private. Some discipline teaching plans were analyzed, according to documentary analysis. The data were collected in the second semester of 2018. Results: the theoretical-practical and methodological curricular activities related to mental health education are inserted in an articulated way with other knowledge fields. This injunction shows that the theoretical dimension has been accompanying the transformations in this field of knowledge and practice. Conclusion: the disciplines include approaches that are coherent with social, economic and cultural situations, but there is little information about the methodological dimension related to the scenarios of practice for mental health education.

Descriptors: Health policy; Higher education institutions; Mental health; Nursing Education; Unique Health System

Resumen: Objetivo: identificar las actividades curriculares teórico-prácticas y metodológicas en la formación del enfermero relacionadas con la educación en salud mental. Método: estudio documental, descriptivo y exploratorio, de enfoque cualitativo. Fue desarrollado en dos Cursos de Graduación en Enfermería de un municipio del interior del Estado del Rio Grande do Sul, siendo uno de ellos de carácter público y otro, privado. Se analizaron los planes de enseñanza de disciplinas, segundo análisis documental. Los datos fueron recogidos en el segundo semestre de 2018. Resultados: las actividades curriculares teórico-prácticas y metodológicas relacionadas al a educación en salud mental están insertadas de modo articulado a otros saberes, mostrando que la dimensión teórica viene acompañando las transformaciones en ese campo de saberes y prácticas. Conclusión: las disciplinas contemplan enfoques coherentes con las situaciones sociales, económicas y culturales, pero se observan pocas informaciones sobre la dimensión metodológica, relacionada con los escenarios de prácticas para la educación en salud mental.

Descriptores: Educación en enfermería; Instituciones de educación superior; Política de salud, salud mental; Sistema único de salud

\section{Introdução}

Os avanços na política de saúde mental, após a criação do Sistema Único de Saúde (SUS), redirecionaram o cuidado aos usuários e suas famílias. Há aproximadamente duas décadas, observa-se o crescimento e a busca por fortalecimento de um modelo diferenciado de cuidado e proteção. ${ }^{1}$ Esse novo modelo de atenção à saúde mental tem repercutido em ações concretas, especialmente com a criação dos Centros de Atenção Psicossocial (CAPS), nos quais se objetiva o atendimento integral, universal e equânime em saúde mental. A partir dessas mudanças, novas formas de organização dos serviços de saúde mental vêm sendo construídas, repercutindo na qualidade de vida dos usuários do SUS. ${ }^{2}$

Embora esses avanços sejam reconhecidos e, comprovadamente, impactem nas ações de cuidado, ainda são observados inúmeros desafios relacionados aos cenários políticos, sociais e 
3 | Silva FP, Siqueira DF, MRCT Marchiori, Colomé JS

econômicos do país, além da dificuldade de superação de um modelo institucionalizado de atenção em saúde mental, caracterizado pela discriminação e segregação dos usuários na sociedade. Mesmo que a universalidade represente um princípio fundamental do SUS, de acordo com a característica de cada território, é evidente a existência de uma conjuntura política atual em acelerado retrocesso, dificultando o avanço contínuo das políticas de saúde no Brasil. ${ }^{3}$

Nesse panorama, a formação dos profissionais para a atuação no SUS precisa acompanhar as transformações que se processam nesse campo de saberes e práticas, de modo ampliado, além das mudanças nas políticas de saúde mental, bem como suas demandas, crescentes e contínuas, de reorganização. Por se tratar de um cenário caracterizado pela multidimensionalidade do cuidado, a saúde mental integra a formação dos profissionais de saúde nos mais diversos níveis. A Enfermagem, nesse contexto, tem atuado de modo integrado a outras profissões e experimenta, atualmente, um processo de transição face às novas Diretrizes Curriculares Nacionais (DCN) para a formação do enfermeiro. ${ }^{4}$

Desse modo, as Instituições de Ensino Superior (IES) devem orientar sua matriz curricular, materializada nos Projetos Pedagógicos dos Cursos de Graduação em Enfermagem, de modo que esses acompanhem as transformações das ações em saúde, no âmbito do SUS. Reiterando o exposto, as DCN preconizam uma sólida formação básica, na qual o estudante seja preparado para enfrentar os desafios das rápidas modificações da sociedade, das condições do exercício profissional e do mercado de trabalho. Para tanto, o processo formativo deve ser desenvolvido em áreas ou núcleos de competência regulados por marcos teóricos e metodológicos que considerem as necessidades coletivas e individuais da população, considerando os vários aspectos que compõem a pluralidade e singularidade do ser humano. ${ }^{4}$

Essas discussões fomentadas pelo processo de construção e reconstrução das DCN, têm incentivado os Cursos de Graduação em Enfermagem em implementar processos formativos por meio de metodologias ativas de ensino e aprendizagem, tanto nos campos teóricos, quanto 
Abordagens teórico-práticas e metodológicas na formação em enfermagem para a saúde... | 4

práticos. Esse modelo formativo busca superar a abordagem direcionada à doença e à transmissão de conhecimentos, apreciando o dialogar e a subjetividade dos atores envolvidos na construção educativa. Para que isso ocorra, há de se considerar estratégias pedagógicas nas quais o educador e o educado integrem, de forma sistemática, o conjunto dos diferentes saberes, não a fragmentar discussões, mas a considerar a importância da integração das reflexões entre ambos protagonistas, no atual cenário social. ${ }^{5}$

Mesmo com o fomento das DCN e os avanços nas políticas de saúde com enfoque no cuidado integral, ainda se tem observado processos de formação reducionistas que ocorrem em cenários de práticas que não tem acompanhado esse contexto ampliado de transformações. Assim, considerando a existência de currículos formais e currículos ocultos nos processos de ensino e aprendizagem, a análise permanente dos modos de se aprender e se ensinar em saúde mental, faz-se pertinente e necessária.

Diante disso, tem-se a seguinte questão de pesquisa: como está organizada a educação em saúde mental nos cursos de graduação em enfermagem, considerando as transformações deste campo de saberes e práticas no SUS? Para tanto, tem-se como objetivo identificar as atividades curriculares teórico-práticas e metodológicas na formação do enfermeiro relacionadas à educação em saúde mental.

\section{Método}

Esse artigo integra uma dissertação de mestrado acadêmico desenvolvida na Área de Concentração Ciências da Saúde e da Vida. Trata-se de um estudo documental, de natureza descritiva e exploratória, estruturado em uma abordagem qualitativa. Os cenários foram os Cursos de Graduação em Enfermagem de duas IES de um município do interior do Estado do Rio Grande do Sul, sendo uma delas de caráter público e outra, privado. A escolha por estas Instituições justifica-se em função destas ofertarem a formação profissional em nível de 
5 | Silva FP, Siqueira DF, MRCT Marchiori, Colomé JS

graduação e pós-graduação. Destaca-se que a pesquisa não teve a intenção de realizar uma análise comparativa dos dois cursos, mas sim ampliar as possibilidades de obtenção de dados em mais de uma realidade concreta.

Foram incluídos na pesquisa os planos de ensino, de ambas as instituições, que contivessem determinados descritores expressos nos seguintes itens: objetivos, ementa, conteúdo programático, metodologia de ensino e avaliação de aprendizagem. Os planos de ensino são compreendidos como instrumentos de organização pedagógica das disciplinas, utilizados no decorrer dos semestres letivos. Possuem estrutura flexível, porém, minimamente, precisam conter os objetivos, ementas, metodologia e avaliação da aprendizagem, além das bibliografias básicas e complementares das disciplinas curriculares.

No que se refere aos descritores mencionados, estes foram selecionados na página de Descritores em Ciências da Saúde (DeCS) - http://decs.bvs.br/, e estão apresentados a seguir: 1. Saúde Mental; 2 Atenção Psicossocial; 3. Centro de Atenção Psicossocial; 4. Redes de Atenção Psicossocial; 5. Psiquiatria/Enfermagem Psiquiátrica; 6. Serviços de Saúde Mental; 7. Clínica Ampliada; 8. Projeto Terapêutico Singular; 9. Reforma Psiquiátrica; 10. Desinstitucionalização; 11. Redes de Atenção à Saúde; 12. Políticas de Saúde; 13. Dependência Química. Portanto, os planos de ensino que continham estes descritores, constituíram o conjunto de documentos analisados.

Destaca-se que foram analisados os planos de ensino de todas as disciplinas dos Cursos de Enfermagem participantes do estudo. Os acessos aos planos de ensino deram-se via Coordenações dos Cursos, as quais disponibilizaram os planos de ensino em formato extensão “PDF” via correio eletrônico. Após a coleta inicial, a partir dos descritores mencionados, foram selecionadas 10 (dez) disciplinas, sendo 5 (cinco) de cada um dos Cursos participantes. Para a análise dos planos de ensino, não houve a distinção entre IES pública ou privada, já que estas foram analisadas de modo conjunto. 
Abordagens teórico-práticas e metodológicas na formação em enfermagem para a saúde... | 6

Os dados foram analisados segundo a análise documental. ${ }^{6}$ Para desenvolver a análise objetivou-se, inicialmente, a constituição de um corpus satisfatório, exaurindo as possibilidades para o alcance de informações relevantes. Assim, após uma análise preliminar dos documentos, procedeu-se à análise documental propriamente dita, reunindo-se todas as partes: elementos da problemática ou do quadro teórico, contexto, autores, interesses, confiabilidade, natureza do texto e conceitos-chave. Nesse processo, buscou-se realizar a interpretação dos dados e sua relação com a literatura, por meio da análise de conteúdo documental. ${ }^{6}$ Essas etapas da pesquisa foram realizadas após a aprovação do estudo em Comitê de Ética em Pesquisa, parecer no 2.457.331.

\section{Resultados e discussão}

A análise de dados resultou em duas categorias temáticas intituladas: "Abordagens teórico-práticas e sua relação com o campo da saúde mental" e "Abordagens metodológicas da formação do enfermeiro para a saúde mental". Os resultados foram apresentados por meio de quadros, seguidos da discussão, conforme apresentado a seguir:

\section{Abordagens teórico-práticas e sua relação com o campo da saúde mental}

A primeira categoria temática foi construída com base nos dados oriundos das ementas, que formam o conjunto de conteúdos programáticos, e dos objetivos das disciplinas selecionadas, apresentados no Quadro 1. 
Quadro 1 - Disciplinas, Ementas e Objetivos dos Planos de Ensino selecionados para o estudo

\begin{tabular}{|c|c|}
\hline Disciplinas & Ementa (E) e Objetivos (O) \\
\hline $\begin{array}{l}\text { D1. Atenção Integral à } \\
\text { Saúde Mental }\end{array}$ & $\begin{array}{l}\text { E: Reforma Psiquiátrica Brasileira. Modelos de atenção psicossocial. Redes de atenção } \\
\text { integral em saúde mental. Ações de promoção, de prevenção, de recuperação e de } \\
\text { reabilitação em saúde mental. Transtornos mentais. Sofrimento psíquico. Reinserção } \\
\text { social e resgate da autonomia. Tecnologias assistenciais em saúde mental. Processo de } \\
\text { cuidado de enfermagem em saúde mental em âmbito individual, familiar e comunitário. } \\
\text { O: Possibilitar o aprendizado crítico-reflexivo e desenvolver o pensamento crítico do } \\
\text { acadêmico em relação aos cuidados de enfermagem no contexto da saúde/doença mental } \\
\text { e suas repercussões e desafios em termos individuais, familiares, comunitários e } \\
\text { coletivos; oportunizar ao acadêmico a participação em equipe multidisciplinar de saúde. }\end{array}$ \\
\hline $\begin{array}{c}\text { D2.Cuidado de } \\
\text { Enfermagem ao Adulto } \\
\text { I }\end{array}$ & $\begin{array}{l}\text { E: Processo de cuidado ao adulto. Segurança do paciente. Clínica Ampliada. } \\
\text { Fisiopatologias dos sistemas. Sistematização da Assistência de Enfermagem } \\
\text { O: Oferecer oportunidade ao acadêmico de enfermagem que propicie uma conduta } \\
\text { centrada nos princípios de valorização humana, responsabilidade e formação de líderes e } \\
\text { profissionais; desenvolver o relacionamento interpessoal entre discente/docente com a } \\
\text { equipe multiprofissional e a clientela, observando princípios éticos específicos do código } \\
\text { de ética de enfermagem. }\end{array}$ \\
\hline $\begin{array}{c}\text { D3. Cuidado em } \\
\text { Enfermagem na Saúde } \\
\text { Mental }\end{array}$ & $\begin{array}{l}\text { E: História da saúde mental e Reforma Psiquiátrica; Modelos de atenção em saúde } \\
\text { mental; Enfermagem em saúde mental e cuidado à família; O: Exame das funções } \\
\text { mentais; Intervenções em saúde mental. } \\
\text { Geral: proporcionar formação acadêmica com vivências práticas voltadas ao processo de } \\
\text { Reforma Psiquiátrica Brasileira. }\end{array}$ \\
\hline $\begin{array}{l}\text { D4. Enfermagem na } \\
\text { Atenção Básica } \\
\text { Rede de Atenção à } \\
\quad \text { Saúde }\end{array}$ & $\begin{array}{l}\text { E: Rede de Atenção à Saúde do Sistema Único de Saúde; Modelos assistenciais em saúde; } \\
\text { Trabalho em equipe; Controle Social e a participação comunitária. } \\
\text { O: Conhecer a organização da rede que integra os serviços do SUS; analisar os modelos } \\
\text { tecnoassistenciais em saúde, identificando a atuação do trabalhador em saúde nas } \\
\text { mudanças necessárias; conhecer e atuar no cuidado de enfermagem no âmbito das } \\
\text { práticas de saúde na AB, envolvendo clínica, epidemiologia e educação em saúde. }\end{array}$ \\
\hline $\begin{array}{l}\text { D5. Enfermagem no } \\
\text { Contexto do Adulto em } \\
\text { Situações Críticas de } \\
\text { Saúde }\end{array}$ & $\begin{array}{l}\text { E: A inserção do acadêmico nos diferentes cenários do cuidado ao adulto; assistência de } \\
\text { enfermagem ao adulto com; traumatismos em geral; enfermagem no cuidado ao adulto } \\
\text { em unidade de internação cirúrgica; enfermagem no cuidado ao adulto em unidade de } \\
\text { centro cirúrgico e sala de recuperação anestésica no contexto hospitalar; enfermagem no } \\
\text { cuidado ao adulto em unidade de terapia intensiva; cuidado em enfermagem na saúde } \\
\text { mental em unidade de internação (enfermagem no cuidado ao adulto sofrimento } \\
\text { psíquico). } \\
\text { O: Cuidar o adulto em situações mais complexas, de forma sistematizada, no } \\
\text { atendimento de suas necessidades pautadas nos princípios éticos e humanísticos, } \\
\text { considerando o ser humano e suas relações no contexto social, político, econômico e } \\
\text { cultural em que está inserido. }\end{array}$ \\
\hline
\end{tabular}


Abordagens teórico-práticas e metodológicas na formação em enfermagem para a saúde... $\mid 8$

\begin{tabular}{|c|c|}
\hline Disciplinas & Ementa (E) e Objetivos (O) \\
\hline $\begin{array}{l}\text { D6. Enfermagem a as } \\
\text { Políticas Públicas }\end{array}$ & $\begin{array}{l}\text { E: História da política de saúde no Brasil e o Sistema Único de Saúde; processo saúde } \\
\text { doença e seus determinantes; promoção da saúde como orientador das práticas em } \\
\text { saúde; a família como unidade de cuidado à saúde } \\
\text { O: Conhecer o sistema de saúde no Brasil; identificar os determinantes socioeconômicos, } \\
\text { ambientais e culturais do processo saúde-doença. }\end{array}$ \\
\hline $\begin{array}{l}\text { D7. Fundamentos } \\
\text { teórico-filosóficos da } \\
\text { Enfermagem }\end{array}$ & $\begin{array}{l}\text { E: Princípios filosóficos e fundamentos teóricos do cuidar em saúde e enfermagem, em } \\
\text { âmbito individual e coletivo. Concepção sistêmica de ser humano, de cuidado de } \\
\text { enfermagem e de saúde ampliada. Saúde e espiritualidade. Aspectos teóricos, filosóficos, } \\
\text { metodológicos e legais que fundamentam a Sistematização da Assistência de } \\
\text { Enfermagem. } \\
\text { O: Desenvolver habilidades crítico-reflexivas e criativas para o exercício competente da } \\
\text { prática clínica ampliada de enfermagem e a tomada de decisões éticas, em âmbito } \\
\text { individual, familiar e comunitário. }\end{array}$ \\
\hline $\begin{array}{l}\text { D8. Gestão dos serviços } \\
\text { de saúde }\end{array}$ & $\begin{array}{l}\text { E: Teorias gerais da administração; Redes de Atenção à Saúde; instrumentos para } \\
\text { gerenciar o cuidado de enfermagem nos processos de trabalho em saúde e atuar nos } \\
\text { problemas nos serviços de saúde e de enfermagem; estrutura física e organizacional dos } \\
\text { serviços de saúde e de enfermagem; política de gestão de pessoas em saúde e } \\
\text { enfermagem; sistema de avaliação e monitoramento; marketing em saúde e enfermagem. } \\
\text { O: Atuar como gerente e líder da equipe de Enfermagem e de serviços de saúde, } \\
\text { participação do planejamento, organização, avaliação e gestão de recursos, objetivando } \\
\text { atender as reais necessidades de saúde da população a partir do perfil epidemiológico e } \\
\text { ampliando a sua participação no processo de gestão. }\end{array}$ \\
\hline $\begin{array}{l}\text { D9. Políticas Públicas } \\
\text { em Saúde }\end{array}$ & $\begin{array}{l}\text { E: Políticas de saúde, saúde pública e saúde coletiva. Articulações entre as políticas } \\
\text { públicas de saúde e as necessidades individuais e coletivas da população. Análise e } \\
\text { evolução do processo histórico das políticas públicas de saúde do Brasil. Reforma } \\
\text { sanitária brasileira. Sistema Único de Saúde - SUS. Controle Social em Saúde. Processo } \\
\text { de construção das políticas de saúde. Políticas Públicas em Saúde vigentes no contexto } \\
\text { brasileiro. } \\
\text { O: Conhecer as políticas públicas em saúde vigentes no contexto brasileiro e suas } \\
\text { implicações nas práticas assistenciais, na organização dos serviços e na gestão de } \\
\text { programas e estratégias articuladas às necessidades individuais e coletivas de saúde da } \\
\text { população. }\end{array}$ \\
\hline D10. Saúde & $\begin{array}{l}\text { E: Conceito ampliado de saúde. Determinantes e condicionantes sociais. } \\
\text { Vulnerabilidades sociais. Processo saúde e doença. Sistemas políticos vigentes no Brasil. } \\
\text { Modelos de atenção à saúde. Temáticas emergentes de saúde e sociedade. } \\
\text { O: Criar situações que propiciem aos acadêmicos desenvolver a capacidade de analisar } \\
\text { de maneira crítica, situações éticas do cotidiano. }\end{array}$ \\
\hline
\end{tabular}

Conforme os dados apresentados, na sequência, as abordagens teórico-práticas estão relacionadas, de modo predominante, ao contexto histórico da saúde mental, à organização da saúde mental no SUS e à inserção da Enfermagem nessa dimensão. Assim, a atual configuração 
da saúde mental, no Brasil, foi relacionada nos planos de ensino estudados, desde uma perspectiva histórica, até se chegar aos arranjos assistenciais presentes nas práticas atuais. Para tanto, os termos analisados denotam que a saúde mental acompanhou as inúmeras transformações evidenciadas nos contextos políticos, econômicos e sociais, culminado com a universalização do acesso em saúde, especialmente a partir da Constituição Federal do Brasil de 1988. Portanto, nos planos de ensino (D6, D9), observou-se, de modo predominante, o termo política de saúde, reiterando a importância do estudo sobre a mesma.

Nessa dimensão, especialmente o movimento sanitário brasileiro integrou as disciplinas e, nesse sentido, considera-se que enquanto processo político e social, esse deve ser questionado quanto aos seus limites e potencialidades, como constitucional e institucional, além de condição essencial para a implantação do SUS no país. ${ }^{3}$ Portanto, para que os limites ainda presentes no conjunto das ações em saúde sejam superados, a reforma precisa ser compreendida como movimento atual, que busca, de modo permanente, possibilidades para a construção e reconstrução do sistema de saúde.

Da mesma forma, o termo SUS esteve mencionado em alguns planos de ensino (D4, D6, D9), denotando que o processo de ensino-aprendizagem do enfermeiro tem acompanhado as discussões que ocorrem na construção da maior política de saúde brasileira, regulamentada na Lei Orgânica 8080/90. O controle social, nessa mesma perspectiva, assegurado por meio da Lei 8.142/90, legitima a discussão sobre a participação da comunidade nas três esferas governamentais de gestão do sistema. ${ }^{7}$

A história das políticas de saúde no Brasil, o processo saúde doença e seus determinantes e sua relação com o SUS, também foram termos observados em diferentes seções dos planos de ensino (D4, D6, D9 e D10) que demonstram ser abordados na formação do enfermeiro. O SUS, organizado de maneira estrutural e sistêmica em todo território brasileiro, oferta suporte às ações de atenção à saúde, viabilizando os princípios e diretrizes dessa política. Além disso, 
Abordagens teórico-práticas e metodológicas na formação em enfermagem para a saúde... $\mid 10$

compreende o conjunto de serviços e ações de saúde, articulados em redes nas três esferas governamentais, possibilitando iniciativas de promoção, vigilância e atenção à saúde..$^{7-8}$

As transformações em saúde no Brasil foram discutidas, assim como alguns modelos de atenção à saúde instituídos e que deveriam ser revistos, de acordo com a atual organização proposta pelo SUS. Com isso, a saúde mental, durante o período do movimento sanitário nos anos 1970/80, recebeu incentivo direto de indivíduos e organizações dispostos a discutir e alterar as práticas de cuidado dos sujeitos com transtornos mentais e uso de drogas. Desse modo, visando proteger indivíduos com transtornos mentais, além de garantir seus direitos, a Reforma Psiquiátrica Brasileira (RPB) buscou transformar o modelo assistencial de saúde mental por meio da Lei 10.216/2001, com enfoque no cuidado e reabilitação dos usuários, sem discriminação de qualquer espécie. ${ }^{1}$

A partir desse cenário, questionaram-se os saberes, as práticas psiquiátricas e o modelo hospitalocêntrico como forma de tratamento. ${ }^{9}$ Com isso, a RPB, de acordo com as ementas e objetivos, reitera a relevância do assunto para a construção acadêmica. Esse novo campo de atores sociais, intersetoriais, múltiplos e articulados com diversos dispositivos e estratégias de inclusão social envolvidos no movimento antimanicomial, formatou uma nova reflexão e discurso sobre a saúde mental, rompendo com a dominante visão da psiquiatria brasileira. ${ }^{10}$

Como um dos produtos da Reforma Psiquiátrica, o termo saúde mental descrito em alguns planos de ensino (D1, D2, D5), reforça as mudanças no pensar, cuidar e fazer saúde de maneira inclusiva, preconizando a cidadania dos usuários, bem como ações com características interdisciplinares, transdisciplinares e intersetoriais. ${ }^{10}$ Nessa visão, o emprego do termo saúde mental, permeando disciplinas mais específicas ao tema, parece denotar uma educação direcionada ao cuidado integral e universal, ampliando as possibilidades de atuação para a Enfermagem, no âmbito do SUS.

Com isso, as IES parecem estar acompanhando as atuais configurações das políticas de saúde no país, desenvolvendo a educação profissional por meio de processos crítico-reflexivos, 
11 | Silva FP, Siqueira DF, MRCT Marchiori, Colomé JS

propostos nos objetivos, buscando desenvolver competências para a gestão, o trabalho em equipe multiprofissional, enfatizando a atenção básica em saúde, com enfoque na participação social. Ainda, as redes de atenção à saúde (RAS), bem como, a clínica ampliada e projeto terapêutico singular (PTS), foram abordados a partir da discussão sobre práticas transformadoras de cuidado mais inclusivas à sociedade (D2, D4, D7 e D8).

Embora o termo atenção básica em saúde tenha sido abordado em menor escala (D4), compreende-se que no contexto das redes de atenção e das políticas de saúde, citadas fortemente, existe uma vinculação direta a esse campo de atuação e de cuidado em saúde. Ademais, compreende-se a atenção básica como o conjunto de ações individuais, familiares e coletivas, sendo a principal porta de entrada e centro de comunicação, além de coordenadora do cuidado, conforme preconizado no referencial das RAS. ${ }^{8}$

Esses dispositivos de cuidado são fundamentais no processo de formação do enfermeiro, a fim de que seja possível agregar diferentes saberes na construção do conhecimento, estabelecendo pontes entre a clínica ampliada e o processo de enfermagem, além de integrar as dimensões cuidado, trabalho e educação. ${ }^{11}$ Nessa esfera de lutas constantes, a saúde mental, como parte do cuidado à saúde, integra-se à atual organização do sistema, estando implicada diretamente nos fluxos assistenciais das redes de atenção à saúde.

A articulação entre a Enfermagem e o campo de saúde mental foi evidenciada especialmente por meio dos termos processo de cuidado ao adulto, enfermagem em saúde mental e o cuidado a família (D1, D2, D3 e D5). Esses assuntos foram articulados a temas como o pensamento crítico, a compreensão da dinâmica dos territórios e o estudo de estratégias de reorganização dos serviços de saúde, denotando preocupação em uma análise estratégica para o planejamento do cuidado em saúde mental.

Os princípios éticos do código de enfermagem foram observados (D2, D5, D7), como também a necessidade de inserção do enfermeiro na equipe multiprofissional (D1, D2, D4), reforçando uma 
Abordagens teórico-práticas e metodológicas na formação em enfermagem para a saúde... $\mid 12$

característica essencial da saúde mental, a atuação interprofissional. Essa necessidade é enfatizada nas DCN, quando essas orientam que os currículos devam buscar um perfil acadêmico e profissional com competências, habilidades e conteúdos que os permitam atuar em equipes interprofissionais com qualidade, eficiência e resolutividade no campo do SUS. ${ }^{4}$ Entendendo que os conhecimentos se integram, a construção grupal e dialogada se faz imprescindível para estruturação de um modelo educacional que busca a integração entre as diversas profissões. ${ }^{5,12}$

\section{Abordagens metodológicas da formação do enfermeiro para a saúde mental}

A segunda categoria temática foi construída com base nos dados oriundos das metodologias de ensino e avaliações da aprendizagem previstas nas disciplinas selecionadas, apresentadas no Quadro 2.

Quadro 2 - Disciplinas, Metodologias de Ensino e Avaliação da Aprendizagem dos Planos de Ensino selecionados para o estudo

\begin{tabular}{|c|c|}
\hline Disciplina & Metodologia de Ensino \\
\hline $\begin{array}{l}\text { D1. Atenção Integral à } \\
\text { saúde Mental }\end{array}$ & $\begin{array}{l}\text { Metodologia da problematização, através do Arco de Maguerez. Avaliação contínua, } \\
\text { cumulativa e compreensiva, em que o acadêmico deverá contemplar os aspectos de ser, } \\
\text { saber e fazer. No cenário de ensino-aprendizagem teórico através de trabalho em grupo, } \\
\text { apresentação e debate de artigos científicos, seminário e provas, participação e } \\
\text { contribuição durante as aulas, responsabilidade no cumprimento de datas e horários, } \\
\text { capacidade de diálogo e de relacionamento com os pares e o docente; frequência às } \\
\text { aulas, capacidade de relacionar a teoria à prática e a composição de um portfólio clínico } \\
\text { reflexivo. }\end{array}$ \\
\hline $\begin{array}{c}\text { D2. Cuidado de } \\
\text { Enfermagem ao Adulto }\end{array}$ & $\begin{array}{l}\text { Atividades teórico-práticas, aulas teóricas com participação ativa dos estudantes sob a } \\
\text { forma expositiva dialogada, recursos audiovisuais, trabalhos e grupos e individuais, } \\
\text { seminários, roteiro de conteúdo, simulação com demonstração prática de alguns } \\
\text { assuntos para posterior aplicação. A disciplina é integrada com as demais disciplinas do } \\
\text { semestre que ocorre por meio das Ações Extensionistas na Atenção Integral à Saúde da } \\
\text { Família I. Na avaliação teórico-prática será considerado o interesse e participação, } \\
\text { responsabilidade, frequência e postura, assim como resultado da aplicação de provas } \\
\text { objetivas/subjetivas, apresentação de trabalhos individuais e em grupo. Na atividade } \\
\text { teórico-prática, o professor supervisor, realizará momentos de reflexão com os alunos } \\
\text { sobre o processo ensino aprendizado e também realizará uma prova prática. }\end{array}$ \\
\hline $\begin{array}{c}\text { D3. Cuidado em } \\
\text { Enfermagem na Saúde } \\
\text { Mental }\end{array}$ & Não informado. \\
\hline
\end{tabular}




\begin{tabular}{|c|c|}
\hline Disciplina & Metodologia de Ensino \\
\hline $\begin{array}{l}\text { D4. Enfermagem na } \\
\text { Atenção Básica }\end{array}$ & $\begin{array}{l}\text { Baseada em metodologias ativas e problematizadoras. Cada professora desenvolverá o } \\
\text { conteúdo seguindo os princípios da participação ativa dos(as) acadêmicos(as), integração } \\
\text { ensino-serviço, aproximação com a comunidade e práticas interdisciplinares, } \\
\text { promovendo o aprendizado significativo. Neste modelo metodológico, acadêmicos e } \\
\text { professoras são atores que promovem o protagonismo dos usuários alvo do cuidado. } \\
\text { Avaliações teóricas individuais ( } 20 \%) \text {, trabalho em grupo }(20 \%) \text {, aulas práticas }(60 \%) \text {. } 2^{\circ} \\
\text { bimestre avaliações teóricas individuais (40\%) e aulas práticas (60\%) da nota final. }\end{array}$ \\
\hline $\begin{array}{l}\text { D5. Enfermagem no } \\
\text { Contexto do Adulto em } \\
\text { Situações Críticas de } \\
\quad \text { Saúde }\end{array}$ & $\begin{array}{l}\text { Divisão de grupos para trabalhos, dinâmicas de grupo. Avaliações por meio de provas e } \\
\text { avaliações práticas; caso o aluno não compareça nas avaliações será realizado texto } \\
\text { dissertativo sobre um ponto referente a disciplina contendo introdução, } \\
\text { desenvolvimento e conclusão; deverá ter clareza, coerência interna e fundamentação } \\
\text { teórica; incluir reflexões vinculadas a experiência prática. }\end{array}$ \\
\hline $\begin{array}{l}\text { D6. Enfermagem e as } \\
\text { Políticas Públicas }\end{array}$ & $\begin{array}{l}\text { Aula expositiva dialogada, visitas aos serviços de saúde, dramatização da realidade. } \\
\text { Trabalhos em grupo com a construção da maquete da territorialização dos serviços de } \\
\text { saúde de Santa Maria e das escolas pertencentes ao PSE. Provas de múltipla escolha. }\end{array}$ \\
\hline $\begin{array}{l}\text { D7. Fundamentos } \\
\text { Teórico-Filosóficos da } \\
\text { Enfermagem }\end{array}$ & $\begin{array}{l}\text { Metodologias ativas de construção do conhecimento como leituras individuais e } \\
\text { complementares, aulas expositivas dialogadas, estudos dirigidos em grupos interativos, } \\
\text { dinâmicas de construção e apresentação de trabalhos em grupo, seminários, atividade } \\
\text { teórico práticas na comunidade apresentação de uma prática clínica de enfermagem } \\
\text { norteada pela SAE e fundamentada na Teoria Sistêmica. Avaliação individual e grupal } \\
\text { dos trabalhos científicos realizados em sala de aula; argumentação crítico-reflexivo em } \\
\text { sala de aula; leituras prévias; análise crítica de artigos e livros. }\end{array}$ \\
\hline $\begin{array}{l}\text { D8. Gestão dos Serviços } \\
\text { de Saúde }\end{array}$ & Não informado. \\
\hline $\begin{array}{c}\text { D9. Políticas Públicas } \\
\text { de Saúde }\end{array}$ & $\begin{array}{l}\text { Metodologias ativas de ensino aprendizagem, como narrativas reflexivas, portfólio, } \\
\text { visitas em cenários de práticas, estudos dirigidos, leituras e discussões em grupos, } \\
\text { seminários e aulas interativas. Avaliação processual durante a disciplina com realização } \\
\text { de atividades/exercícios, participação em aula, narrativas reflexivas, evidenciando leitura } \\
\text { e análise crítica acerca dos conteúdos abordados; portfólio e avaliação teórica individual; } \\
\text { seminário temático realizado a partir de visitas e ações estratégicas em cenários de } \\
\text { gestão de políticas de saúde. }\end{array}$ \\
\hline D10. Saúde e Sociedade & $\begin{array}{l}\text { Aulas expositivas dialogadas, estudos dirigidos, leituras e discussões em grupos, } \\
\text { apresentação e debates, sistematização e produção de textos individual e ou em grupos, } \\
\text { com base em textos previamente indicados relativos ao assunto. Práticas junto à } \\
\text { comunidade no município. Trabalhos científicos realizados em grupo; portfólio; } \\
\text { acadêmico, resenhas; críticas; relatórios, sínteses; avaliação individual escrita; dinâmica, } \\
\text { iniciativa e criatividade. }\end{array}$ \\
\hline
\end{tabular}

$\mathrm{Na}$ sequência, as abordagens metodológicas foram organizadas, essencialmente, em metodologias tradicionais de ensino-aprendizagem e, numa visão mais ampla, em metodologias ativas e participativas na formação do enfermeiro. 
Abordagens teórico-práticas e metodológicas na formação em enfermagem para a saúde... | 14

Diante das metodologias de ensino e avaliação de aprendizagem, em uma abordagem mais tradicional, os documentos registraram provas individuais, aula expositiva dialogada com uso de recursos audiovisuais, como slides (D2, D4, D6, D7 e D10). Entende-se que esses recursos também podem ser utilizados de uma forma crítica e reflexiva; porém, foram discutidos em separado, uma vez que alguns referenciais os compreendem como métodos mais tradicionais de ensino. Em algumas situações, esses recursos são entendidos como meios de transmissão de conhecimento, nos quais o estudante assume postura passiva. Para isso, uma perspectiva mais crítica e ampliada deve estar presente nas instituições formadoras, a fim de se alcançar o perfil de competências esperado para o enfermeiro. ${ }^{13}$

Embora a apresentação dos documentos contenha uma construção inovadora, em que o estudante protagoniza seu método investigativo, existe explícita em alguns planos, a indefinição de cenários de atuação, pois não esclarecem a relação teoria/prática, e sim, modos avaliativos fragmentados, pois a abordagem teórica, em alguns casos, se mostra predominante, deixando em segundo plano as vivências práticas/estágios em que o estudante está inserido (D2 e D4).

Em relação às metodologias ativas e participativas de ensino-aprendizagem, algumas disciplinas as destacaram de modo bastante explícito (D1, D4, D7, D9), indo ao encontro das abordagens críticas e emancipatórias, nas quais o estudante é instigado a envolver-se intensamente com o propósito de sua construção, devendo ser uma realidade cada vez mais explorada. Desse modo, nos planos analisados, os portfólios (D1, D9 e D10) foram considerados como ferramentas de aprendizagem inseridas nessa perspectiva, uma vez que proporcionam investigação, autonomia e reflexões transformadoras para o processo educativo.

Contudo, a utilização de portfólios traz consigo inúmeros desafios, como o envolvimento dos estudantes, organização da disciplina, pouco tempo para seu desenvolvimento, carga horária do estudante no semestre, mas, ainda assim, reforça o protagonismo do estudante em sua formação profissional. Portanto, oportuniza uma análise acerca do próprio processo educativo, a 
15 | Silva FP, Siqueira DF, MRCT Marchiori, Colomé JS

partir da discussão das fragilidades e potencialidades, o que poderia ser mais explorado pelo estudante, sugerindo a reelaboração da análise descrita e, com isso, alcançando diferentes patamares de conhecimentos e vivências. ${ }^{14}$

Além dos portfólios, também integraram as metodologias ativas de ensinoaprendizagem, a participação dos estudantes em seminários, na realização de leituras sucedidas de análise crítica dos conteúdos desenvolvidos, no fomento à iniciativa e à criatividade na apresentação dos trabalhos previamente agendados, além de estudos dirigidos, inseridos tanto nas metodologias de ensino, como na avaliação de aprendizagem. Ainda, a utilização da metodologia problematizadora (D1 e D4), indica o emprego de modelos inovadores, proporcionando ao estudante interagir com o conhecimento sistematizado de modo ativo, como ator do seu processo de formação. ${ }^{15}$ Como exemplo de uma abordagem problematizadora nos planos analisados, pode-se citar o Arco de Maguerez (D1), que por meio de um processo sistematizado, denota a preocupação com a construção autônoma do estudante.

Mediante ao apresentado, pode-se considerar que as rápidas transformações, que ocorrem de modo locorregional e global, desafiam as universidades para a utilização de novos métodos de ensino- aprendizagem, contemplando uma nova concepção de mundo, sociedade e homem. ${ }^{16}$ Apoiadas nessa construção, as DCN buscam orientar iniciativas que permitam aos estudantes o aprender a aprender, que envolve ainda, o aprender a ser, aprender a fazer, aprender a viver em comunhão e aprender a conhecer, com vistas à integralidade da atenção em saúde de indivíduos, famílias e comunidades. ${ }^{4}$

Em síntese, as metodologias de ensino e de avaliação da aprendizagem, vem acompanhando as transformações no campo da saúde mental, de maneira a buscar a compreensão do indivíduo no seu contexto social. Essas mudanças requerem dos professores exigências não somente pedagógicas, mas também relacionais e dialógicas para a discussão ampliada desse campo de saberes e práticas junto aos estudantes e profissionais das equipes dos serviços. 
Abordagens teórico-práticas e metodológicas na formação em enfermagem para a saúde... | 16

Como limitação, tem-se a análise específica de documentos de duas instituições formadoras, sendo, portanto, importante a realização de estudos de campo em outros cenários que possam dar voz aos protagonistas do processo de ensino-aprendizagem em saúde mental.

\section{Conclusão}

As atividades curriculares teórico-práticas e metodológicas na formação do enfermeiro relacionadas à educação em saúde mental estão inseridas, na maioria das disciplinas analisadas, de modo integrado e articulado a outros saberes, mostrando que a dimensão teórica vem acompanhando as transformações que ocorrem no contexto estudado. Contudo, a dimensão metodológica dos planos analisados ainda necessita estar mais clara em relação à inserção e às ações dos estudantes nos cenários de saúde mental.

Em relação às metodologias de ensino-aprendizagem, predominaram as ativas e problematizadoras, demonstrando preocupação dos cursos em fomentar uma formação crítica e proativa, articulada à realidade dos cenários, de modo reflexivo e propositivo. Ainda, a inserção na realidade dos serviços traz uma perspectiva formativa que fomenta a articulação do ideal e do real, abrindo possibilidades para novas estratégias em saúde mental.

Em virtude disso, os cursos precisam articular-se de modo integrado à gestão, aos serviços e às equipes de saúde, de modo a pensar e atuar de modo coletivo no enfrentamento das problemáticas que se apresentam nesse campo.

\section{Referências}

1. BRASIL. Ministério da Saúde. Presidência da República. Subchefia para Assuntos Jurídicos. Lei no 10.216, de 06 de abril de 2001. Dispõe sobre a proteção e os direitos das pessoas portadoras de transtornos mentais e redireciona o modelo assistencial em saúde mental. Brasília (DF): Ministério da Saúde, 2001. Disponível em: http://www.planalto.gov.br/ccivil_03/leis/leis_2001/110216. Acesso em: 25 jun. 2017.

2. BRASIL. Ministério da Saúde. Portaria no 366, de 19 de fevereiro de 2002. Brasília, DF: Ministério da Saúde, 2002. Disponível em: http://bvsms.saude.gov.br/bvs/saudelegis/gm/2002/prt0336_19_02_2002. 
Acesso em $10 \mathrm{mar} / 2017$.

3. Cohn A. "Caminhos da reforma sanitária”, revisitado. Estudos Avançados. 2018;32(93):225-41. doi: https://doi.org/10.5935/0103-4014.20180040

4. BRASIL. Ministério da Educação. Conselho Nacional de Saúde. Resolução no 573, de 31 de janeiro de 2018. Diário Oficial da União: Seção 1, Brasília, DF, p. 38., 06 nov. 2018. Disponível em: https://conselho.saude.gov.br/resolucoes/2018/Reso573.pdf. Acesso em: 12 nov. 2018.

5. Soares AN, Souza V, Santos FBO, Carneiro ACLL, Gazzinelli MF. Dispositivo Educação em Saúde: reflexões sobre práticas educativas na atenção primária e formação em enfermagem. Texto Contexto Enferm. 2017;26(03):e0260016. doi: https://doi.org/10.1590/0104-07072017000260016

6. Cellard A. A análise documental. In: Poupart J. A pesquisa qualitativa: enfoques epistemológicos e metodológicos. 3ª ed. Vozes: Rio de Janeiro; 2012. p. 295-316.

7. Vasconcelos CM, Pasche D. O SUS em perspectiva. In: Campos GWS (Org). Tratado de saúde coletiva. $2^{\underline{a}}$ ed. Hucitec: São Paulo; 2012. p. 559-90.

8. Morosini MVGC, Fonseca AF, Lima LD. Política nacional de atenção básica 2017: retrocessos e riscos para o sistema único de saúde. Saúde Debate. 2018;42(116):11-24. doi: https://doi.org/10.1590/01031104201811601

9. Andrade APM, Maluf SW. Experiências de desinstitucionalização na reforma psiquiátrica brasileira: uma abordagem de gênero. Interface Comun Saúde Educ (Online). 2017;21(63):811-21. doi: https://doi.org/10.1590/1807-57622015.0760

10. Amarante P, Torre EHG. Loucura e diversidade cultural: inovação e ruptura nas experiências de arte e cultura da reforma psiquiátrica e do campo da saúde mental no Brasil. Interface Comun Saúde Educ. 2017;21(63):763-4. doi: https://doi.org/10.1590/1807-57622016.0881

11. Rocha EM, Lucena AF. Projeto terapêutico singular de enfermagem em uma perspectiva de cuidado interdisciplinar. Rev Gaúcha Enferm. 2018;24(39):e2017-0057. doi: https://doi.org/10.1590/19831447.2018.2017-0057

12. Magnago C, Pierantoni CR, Carvalho CL, Girardi SN. A formação do enfermeiro em diferentes regiões de saúde do Brasil. Rev Bras Saude Mater Infant (Online). 2017;17(1):S219-228. doi: https://doi.org/10.1590/1806-9304201700s100010

13. Peres CRFB, Marin MJS, Soriano ECI, Ferreira MLSM. Um olhar dialético para as mudanças curriculares na formação do enfermeiro. Rev Esc Enferm USP. 2018;24(52):e03397. doi: https://doi.org/10.1590/s1980-220x2017038003397

14. Macedo KDS, Acosta BS, Silva EB, Souza NS, Beck CLC, Silva KKD. Metodologias ativas de 
Abordagens teórico-práticas e metodológicas na formação em enfermagem para a saúde... $\mid 18$

aprendizagem: caminhos possíveis para inovação no ensino em saúde. Esc Anna Nery Rev Enferm. 2018;22(3):e20170435. doi: https://doi.org/10.1590/2177-9465-ean-2017-0435

15. Vieira MNCM, Panúncio-Pinto MP. A metodologia da problematização (MP) como estratégia de integração ensino-serviço em cursos de graduação na área da saúde. Medicina (Ribeirão Preto). 2018;48(3):241-8. doi: https://doi.org/10.11606/issn.2176-7262.v48i3p241-248

16. Nicola RMS, Behrens MA. Contribuições da teoria da complexidade para inovação no planejamento pedagógico do ensino superior. Rev Diálogo Educ (Online). 2017;17(52):357-86. doi: https://doi.org/10.7213/1981-416X.17.052.DS03

Editor Científico: Tânia Solange Bosi de Souza Magnago

\section{Autor correspondente}

Fabiana Porto da Silva

E-mail: fabianaporto54@gmail.com

Endereço: Conde de Porto Alegre n. 217. Apto.102 Ed. Santorini. Bairro Centro, Santa Maria, Rio Grande do Sul, Brasil.

CEP: 97010100

\section{Contribuições de Autoria}

1 - Fabiana Porto da Silva

Concepção e planejamento do projeto de pesquisa, coleta, análise e interpretação dos dados, redação e revisão crítica.

\section{2 - Daiana Foggiato de Siqueira}

Redação e revisão crítica.

\section{3 - Mara Regina Caino Teixeira Marchiori}

Redação e revisão crítica.

\section{4 - Juliana Silveira Colomé}

Concepção e planejamento do projeto de pesquisa, análise e interpretação dos dados, redação e revisão crítica.

\section{Como citar este artigo}

Silva FP, Siqueira DF, MRCT Marchiori, Colomé JS. Abordagens teórico-práticas e metodológicas na formação em enfermagem para a saúde mental. Rev. Enferm. UFSM. 2020 [Acesso em: Ano Mês Dia]; vol.10 e77: 1-18. DOI:https://doi.org/10.5902/2179769240141 